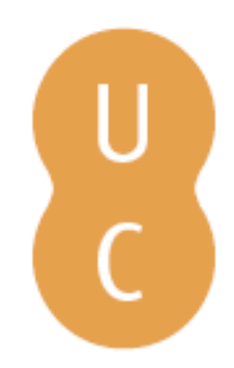

\title{
nombalina
}

\section{Virtù e fortuna nelle Vitae e nei Moralia di Plutarco}

Autor(es): Becchi, Francesco

Publicado por: Imprensa da Universidade de Coimbra; Katholieke Universiteit Leuven

URL

persistente: URI:http://hdl.handle.net/10316.2/32089

DOI: $\quad$ DOI:http://dx.doi.org/10.14195/978-989-26-0462-6_3

Accessed : $\quad$ 26-Apr-2023 06:09:53

A navegação consulta e descarregamento dos títulos inseridos nas Bibliotecas Digitais UC Digitalis, UC Pombalina e UC Impactum, pressupõem a aceitação plena e sem reservas dos Termos e Condições de Uso destas Bibliotecas Digitais, disponíveis em https://digitalis.uc.pt/pt-pt/termos.

Conforme exposto nos referidos Termos e Condições de Uso, o descarregamento de títulos de acesso restrito requer uma licença válida de autorização devendo o utilizador aceder ao(s) documento(s) a partir de um endereço de IP da instituição detentora da supramencionada licença.

Ao utilizador é apenas permitido o descarregamento para uso pessoal, pelo que o emprego do(s) título(s) descarregado(s) para outro fim, designadamente comercial, carece de autorização do respetivo autor ou editor da obra.

Na medida em que todas as obras da UC Digitalis se encontram protegidas pelo Código do Direito de Autor e Direitos Conexos e demais legislação aplicável, toda a cópia, parcial ou total, deste documento, nos casos em que é legalmente admitida, deverá conter ou fazer-se acompanhar por este aviso.

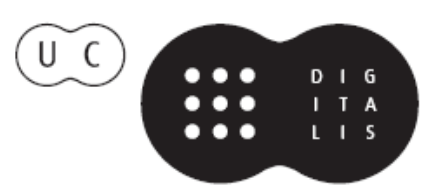




\title{
Puilosophy in Society \\ Virtues and Values in Plutarch
}

\author{
José Ribeiro Ferreira \\ LUC VAN DER STOCKT \\ Maria do Céu Fialho
}

Editors

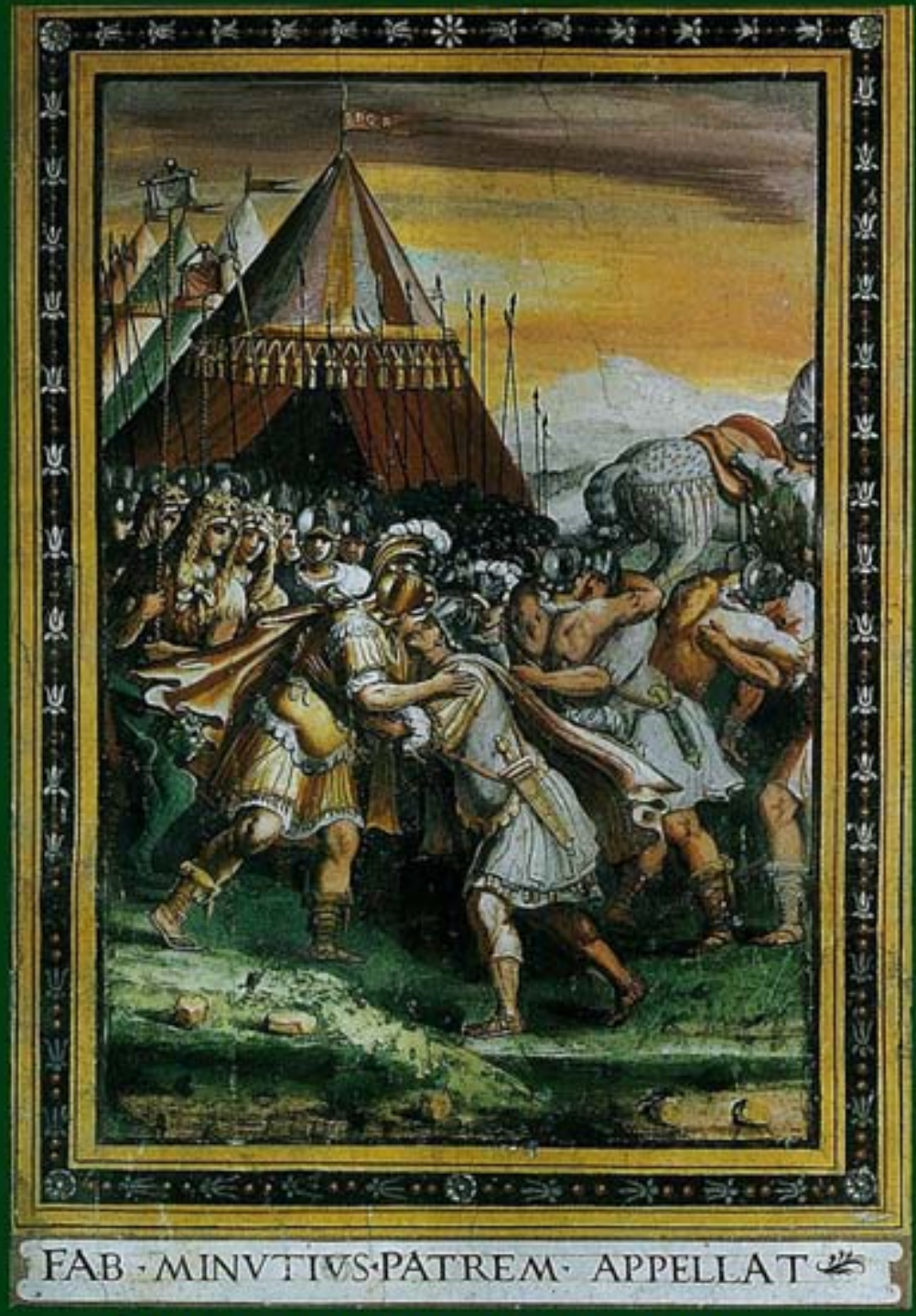

KATHOLIEKE UNIVERSITEIT LEUVEN IMPRENSA DA UNIVERSIDADE DE COIMBRA Leuven-Coimbra, 2008 
(Página deixada propositadamente em branco) 
José Ribeiro Ferreira, Luc van der Stockt \& Maria do Céu Fialho

EDITORS

\section{PhILOSOPHY IN Society}

\section{Virtues and Values in Plutarch}

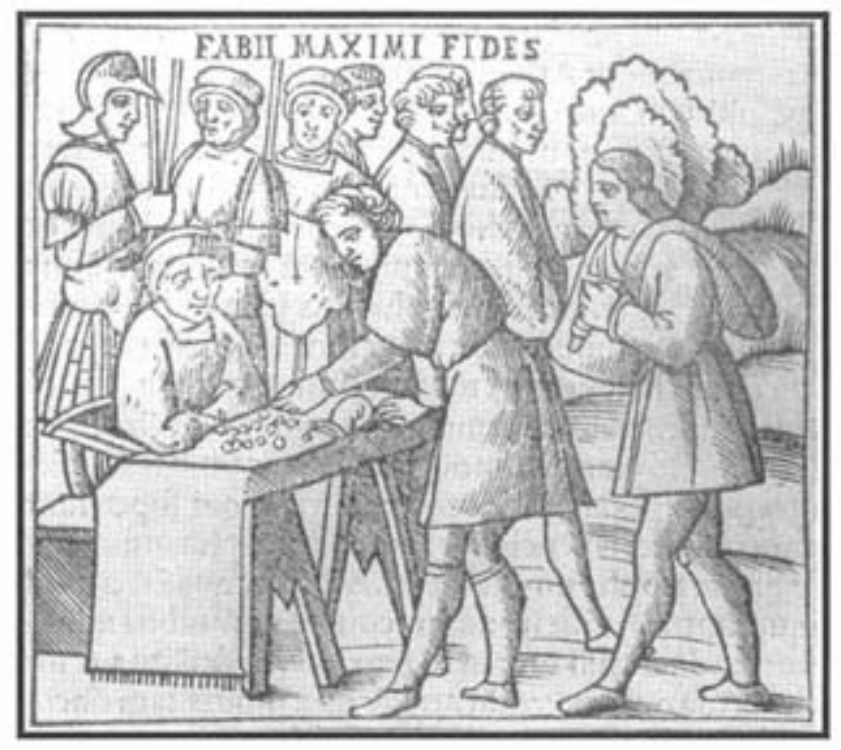

Fabius Maximus' Loyalty

Vitae Plutarchi Cheronei novissime post Jodocum Badium Ascensium longe diligentius repositae maioreque diligentia castigatae, cum copiosiore verioreque indice, nec non cum Aemilii Probi vitis, una cum figuris, suis locis apte dispositis, Venetiis 1516 , fol . 65v

\section{LEUVEN - CoIMBRA \\ 2008}

KATHOLIEKE UNIVERSITEIT LEUVEN

IMPRENSA DA UNIVERSIDADE DE COIMBRA 


\title{
First published 2008
}

\section{(C) Universiteit KATHOlieke Leuven \\ (c) Universidade Coimbra}

\author{
Published by \\ IMPRENSA DA UNIVERSIDADE COIMBRA \\ Imprensa da Universidade de Coimbra \\ Rua da Ilha, $\mathrm{n}^{\circ} 1$ \\ 3000-033 Coimbra (Portugal) \\ Email: imprensauc@ci.uc.pt \\ URL: http://www.uc.pt/imprensa_uc
}

ISBN: 972-989-8074-73-7

Legal Deposit: MA-140-2009

\author{
Printed in Spain by \\ IMAGRAF IMPRESORES, S.A. \\ c/ Nabucco 14 \\ 29006 Málaga \\ Tfno. 952328597
}

\section{Frontispiece:}

Fabius Maximus and Minucius (Francesco da Siena, Grottaferrata, Palazzo Abbaziale). We are grateful to the Archimandrita of the "Monastero Esarchico di Santa Maria di Grottaferrata", P. Emiliano Fabbricatore, for the authorization to reproduce this picture. 


\section{Virtù e fortuna nelle Vitae e nei Moralia di Plutarco \\ Francesco BecCHI Universtrà degli Studi di Firenze}

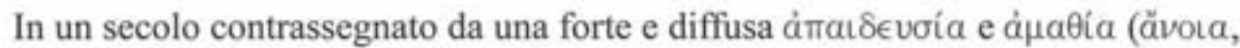

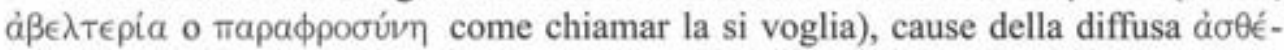

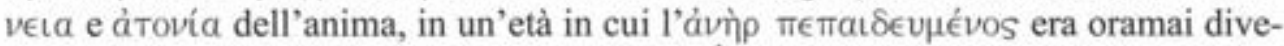
nuto un monstrum, più raro dell'araba fenice', Plutarco per educare l'aristocrazia

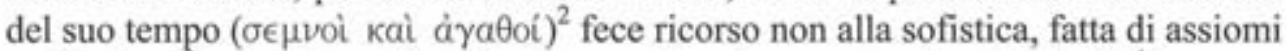
e sillogismi ${ }^{3}$, ma ad una filosofia che non è teoria, ma prassi e arte di vita ${ }^{4}$ in quan-

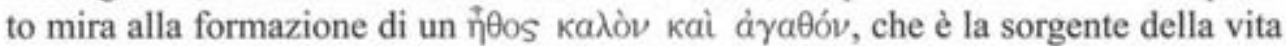
e da cui scaturiscono le belle azioni, che sono la manifestazione esterna di una disposizione interiore virtuosa ${ }^{5}$. Il discorso filosofico infatti non è per Plutarco come la scultura che modella statue che si ergono immobili su un piedistallo, come dice Pindaro, ma a tutto ciò che tocca comunica attività, efficacia, vitalità; ispira il desiderio di agire, i giudizi corretti che generano atti utili, begi ideali, un nobile orgoglio, una grandezza d'animo unita a dolcezza e semplicità.

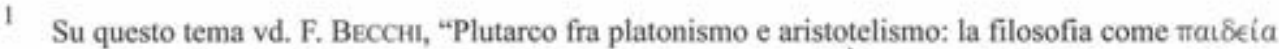
dell'anima", in: A. Pérez Jiménez, J. Garcia López y Rosa M^ Agutlar (eds.), Plutarco, Platón y Aristóteles, Actas del V Congreso Internacional de la I.P.S., Madrid-Cuenca, 4-7 de Mayo de 1999, Madrid, 1999, pp. 27-28.

Plut., bruta anim. 990D.

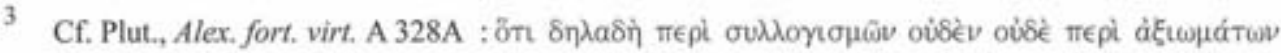

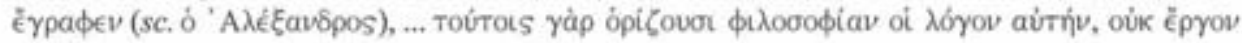

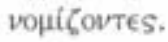

4 Plut., Alex, fort. virt. A 328A; quaest. conv. 1 613B.

Plut., prof. virt. 84C; tranq. an. 477BC.

6

Plut., cum princ. philos. 776C.

Josê Ribeiro Ferreira, Luc Van der Stockt \& Maria do Céu Fial.ho (Edd.), Philosophy in Society - Virtues and Values in Plutarch, Leuven-Coimbra, 2008, pp. 39-52. 
Una filosofia quindi fondata sulla razionalità e sull'affettività che rappresenta il

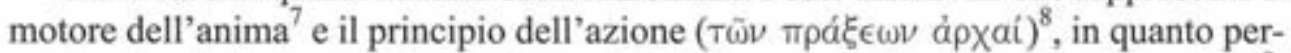
mette alle virtù, dono della ragione e dell'educazione, di trasformarsi in azioni ${ }^{9}$.

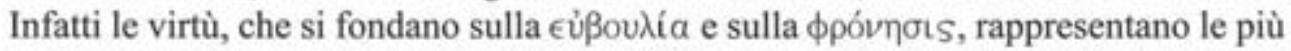

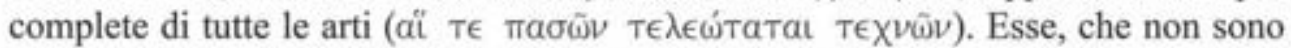
qualcosa di fisso, perché non sono "essere", ma "attività", né sono "sostanza", ma "funzione", si manifestano continuamente nelle opere e nella condotta di ogni gior$\mathrm{no}^{10} \mathrm{e}$ con le loro realizzazioni ci pongono in una condizione tale per cui, diversamente dai beni di fortuna, di cui bramiamo il possesso e l'uso, ammiriamo i fatti e desideriamo imitarne gli autori ${ }^{11}$. Ma le virtù, anche se si risolvono in azioni utili ${ }^{12}$, sono innanzi tutto dei giudizi e delle opinioni corrette sull'onesto, sul giusto, sull'utile come anche sul turpe, sull'ingiusto e sul dannoso ${ }^{13}$. La pratica e l'esercizio della virtù, che è la condizione stessa della felicità e della pienezza dell'essere, pre-

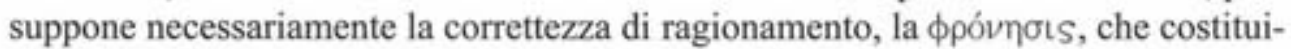
sce l'arte più importante e perfetta e rappresenta il coronamento della buona reputazione e di ogni pretesa umana ${ }^{14}$.

Ragione e passione costituiscono per Plutarco i necessari presupposti dell'agire non solo etico, ma anche civile e politico ${ }^{15}$, il che determina la sua presa di distanza tanto da chi, come gli Stoici, fanno delle passioni dei processi della ragione, come se tutti i desideri e i moti d'ira fossero dei giudizi, quanto da chi, come gli Epicurei ${ }^{16}$, squalificano l'eccellenza della virtù, riducendola ad un fenomeno di natura passionale ${ }^{1 \text { ? }}$.

CH. Froldefond, "Plutarque et le platonisme", $A N R W$ II 36.1, Berlin-New York 1987, p. 202 : "cc'est l'affectivité...qui est le moteur de l'âmen.

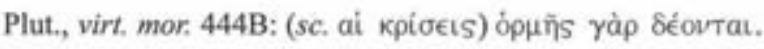

9 Questi giudizi per tradursi in azioni necessitano poi di un impulso che l'ñtos, in quanto qualitả

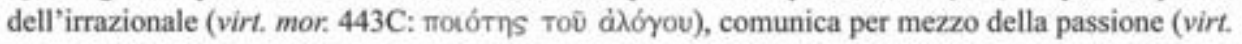
mor. 444B). Sulla figura del vero filosofo vd. Plut., Alex. fort. virt. A 328AB, 333BC.

Plut., Per. 2, 2.

Sulla necessità di trasferire i giudizi alle opere e di non lasciare che i discorsi restino discorsi, ma diventino azioni vd. Plut, prof. virt. 84B; cum princ, philos, $776 \mathrm{C}$.

Plut., Demetr. 1, 4.

Sulla necessità che il giudizio dell'uomo sia consolidato e rafforzato dalla ragione e dallo studio della filosofia vd. Plut., Tim. 6, 1-2.

15 Sulla necessità che nelle azioni politiche potenza e fortuna vadano congiunte con saggezza e giustizia vd. Plut., Dio 1, 3.

16 Plut., suav, viv. Epic. $1101 \mathrm{~A}$.

17

Plut, an. procr. in Tim. 1025D. Per il valore come mancanza di paura (ảфoßía) vd. Plut., Cleom. 9, 3. 
I modelli etici a cui il discepolo di Ammonio si ispira sono quelli propri della cultura classica:

1) il filo sacro del raziocinio che, in quanto d'oro, è pieghevole e segue costantemente la via di mezzo che rifugge dal puro piacere come dal puro dolore ${ }^{18}$, perché la ragione si caratterizza non solo per la sua eccellenza ma anche e soprattutto per la sua mitezza (

2) la virtù, di cui non c'è bene né più grande né più piacevole ${ }^{20}$ né più divino $0^{21}$ di cui amiamo la realizzazione ${ }^{22}$, intesa come correttezza di ragionamento, culmine della natura logica, disposizione dell'anima concorde con se stessa ${ }^{23}$, cioè coerenza e identità con la propria storia ${ }^{24}$ attraverso il passare delle alterne esperienze e vicissitudini ${ }^{25}$ che mettono a nudo pregi e difetti, virtù e vizi di un uomo. Il virtuoso infatti, vivendo con la virtù attraverso le azioni ${ }^{26}$ dà prova di essere non solo coerente con se stesso, ma superiore a se stesso, mentre il malvagio, che è privo di questa identità, dimostra tutta la propria inferiorità.

In questa linea di sviluppo che porta alla formazione di un carattere capace di acquisire e praticare la virtù sia nelle parole che nelle azioni ${ }^{27}$, giuocano un ruolo importante vari fattori come la natura ( $\phi u ́ \sigma \iota s)$, che l'aristocrazia dei primi secoli dell'impero sembra aver trasformato in "natura di ciò che è contro natura" ( $\phi$ vics

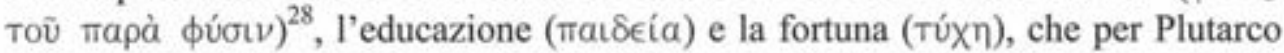
rappresenta la cartina di tornasole per mostrare di che pasta è fatto un uomo.

La $\phi v i \sigma s^{29}$, che tutto genera e produce, ritenuta fortuna dagli epicurei, e sapien-

18

19

Plat., Lg. 793a.

Plat., Lg. 645a.

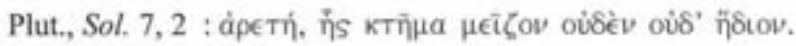

Plut., Arist. 6, 5: ...Tìv $\delta^{\prime}$ à

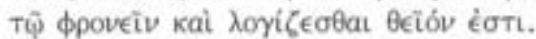

Plut., Per: 1, 3-4.

Plut., and. poet. 24D.

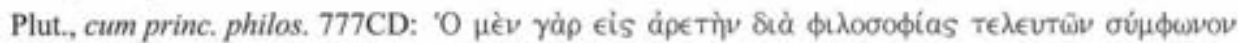

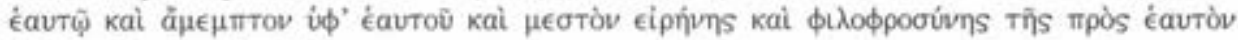

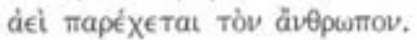

R. BODEI, Geometria delle passioni. Paura, speranza, felicità: filosofia e uso politico, Milano 1994, p. 200.

Plut., prof. virt. $80 \mathrm{E}$.

Plut., tuend. san. 137E.

Plut., tuend. san. 132A.

Non direi con KonRAd (Plutarco, Sertorio-Eumene, Milano 2004, p. 245 n. 80) che «da physis è di scarso interesse per il biografos, ma piuttosto che essa non è eticamente significativa per il filosofo di Cheronea. 
za, cioè, virtù dagli stoici ${ }^{30}$, per Plutarco induce di per sé al bello ${ }^{31}$, ma può anche

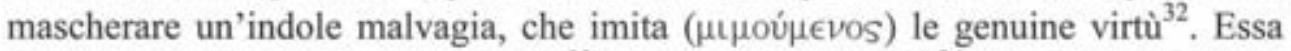
quindi, se generosa e buona ( $\chi \rho \eta \sigma T \eta^{33}$ o $\gamma \in v v a i a$ кà à $\gamma a \theta \eta^{34}$ ), permette alla virtù, come a un pollone forte e resistente, di mettere radici e diventare una pianta vigorosa e resistente ${ }^{435}$. Essa consente all'uomo di essere moderato nella buona sorte e coraggioso nelle avversità ${ }^{36}$, dandogli la forza di imitare ciò che ammira e di fuggire ciò che disapprova, e quindi di dimostrare di non essere né debole (ov̉ taाtє-

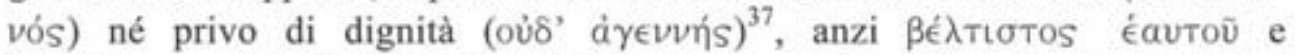

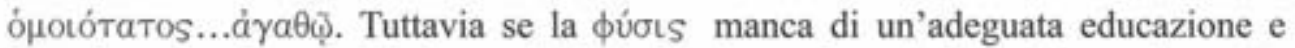

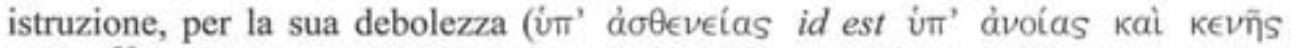

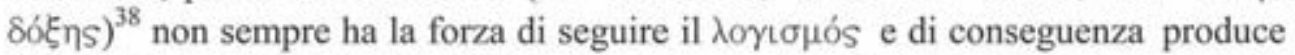
insieme a quelli buoni, risultati cattivi, come avviene in agricoltura di un terreno

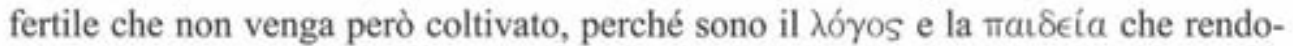
no l'indole naturale capace di accogliere la moderazione e di respingere l'eccesso ${ }^{39}$. Tutti gli uomini sono infatti per natura in grado di formulare retti giudizi proprio perché, come si è detto, la natura di per sé induce al bello, ma ciò per cui gli uomini virtuosi si distinguono è il fatto che essi hanno criteri di giudizio forti e solidi nelle avversita ${ }^{40}$, dove le circostanze della Túx $\eta$ infrangono i ragionamenti ( $\theta$ pav́ou-

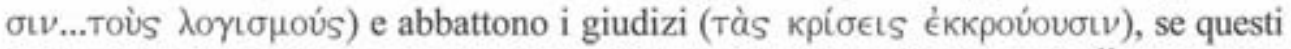
non sono consolidati e rafforzati dalla ragione e dallo studio della filosofia ${ }^{41}$. Non basta infatti che sia nobile e retta l'azione che si intraprende: occorre anche che sia irremovibile il giudizio da cui si parte $e^{42}$. Quindi non solo una natura mediocre che la buona

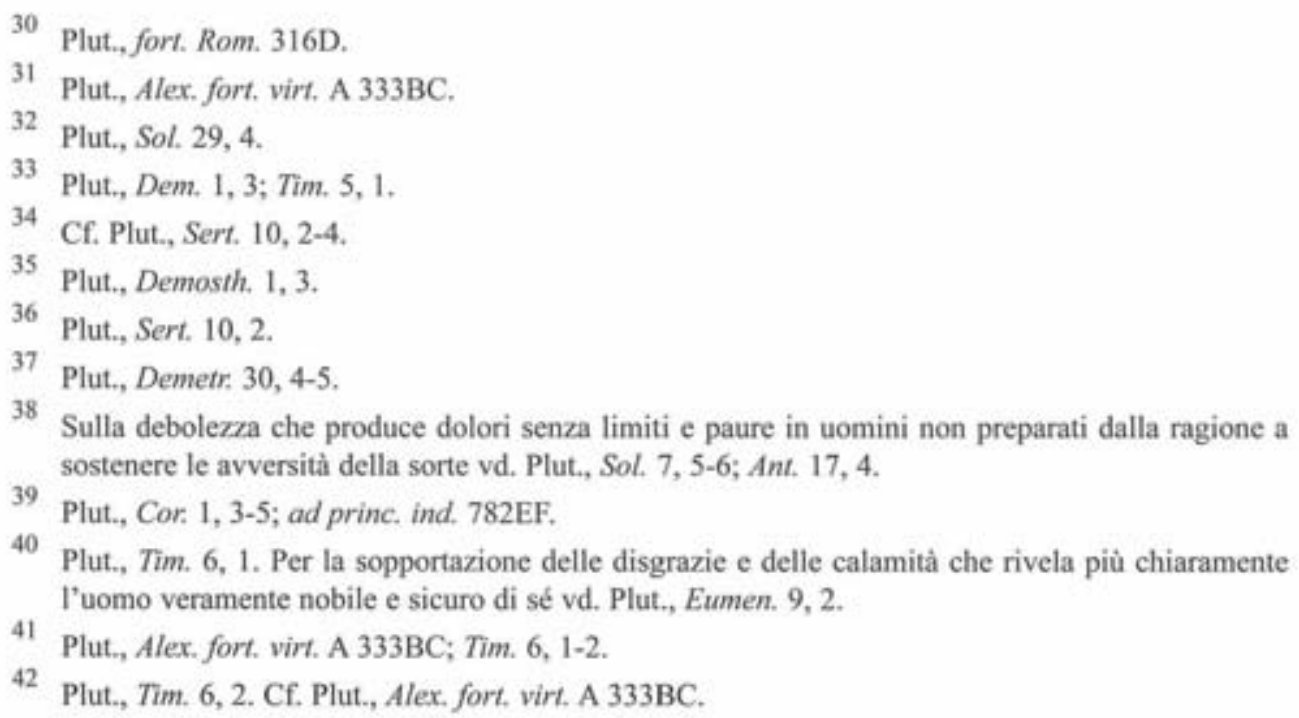


sorte ha elevato ad una posizione privilegiata, conferendole grandezza e maestà ${ }^{43}$, ma

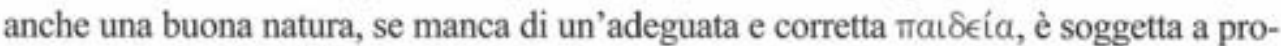
fonde modificazioni ${ }^{44}$, trasformandosi anche nel suo contrario, quando la buona sorte l'abbandona, come capitò a Sertorio che, colpito da grandi e immeritate sventure ${ }^{45}$, non fu in grado di sopportarle con equilibrio e moderazione ${ }^{46}$.

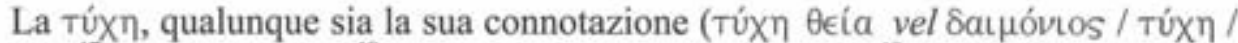

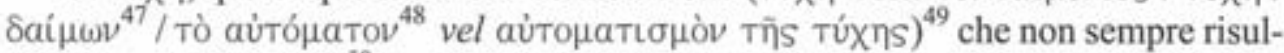
ta agevole distinguere ${ }^{50}$, rappresenta comunque per Plutarco una causa che sfugge

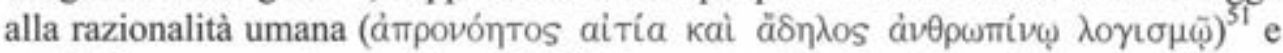

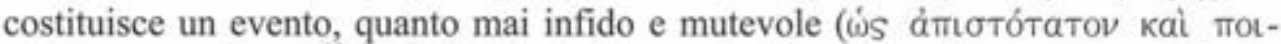

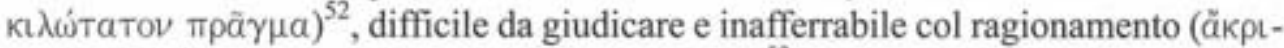

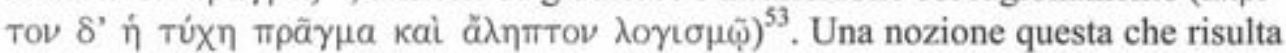
molto vicina a quella che l'Autore della Consolatio ad Apollonium, legato sempre alla cerchia di Plutarco, attribuisce a Teofrasto ${ }^{54}$ :

43

44

45

46

47

49

50

S!

52

53

54

Plut., Eum. 9, 1-2.

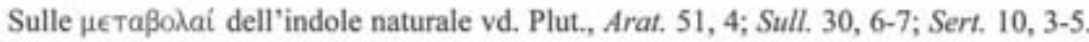

Plut., Sert. 10, 6-7.

Plut., Sert. 10, 2, 5-7; Alc. 2, 1; Alex. 42, 3-4; Sull. 30, 6. Sulla sopportazione delle disgrazie c delle calamità come condizione in cui più chiaramente si manifesta l'uomo veramente nobile $\mathrm{e}$ sicuro di sé, vd. Eum. 9, 2.

Sulla nozione di \&ai $\mu \omega v$ personale che nei Moralia è impiegata per indicare la divinitả, mentre nelle Vitae risulta associata e assimilata a quella di tyche cf. Plut., Alex. 30, 4; 52, 2; Sull. 6, 8; Alc. 33, 2; Tim. 36, 5; Mar. 46, 1; Caes. 38, 5; 69, 2; Ant. 33, 2-3. Sulla demonologia plutarchea vd.: D. H. Russel, Plutarch, London 1972 (1973?), 75-78; F. E. BRENK. S. J., In mist apparelled. Religious Themes in Plutarch's "Moralims and "Lives", Leiden 1977, pp. 49 sgg., 92 sgg., 130 sgg.; G. ForN1, Plutarco, La Fortuna dei Romani, Napoli 1989, pp. 16-20; S. Swarn, "Plutarch: Chanse, Providence, History", AJPh, 110, 1, pp. 272-302; E. A. GARCIA GARCIA, "La idea de daimon en Plutarco", in M. Garcia Valdés (Ed.), Estudios sobre Plutarco: ideas religiosas, Actas del III Simposio Internacional sobre Plutarco (Oviedo, 30 aprile - 2 maggio 1992), Madrid 1994.

Plut., Sert. 1, 1-2.

Vd. F. BECCH, "Túxๆ: storia di un nome", in Studi in onore di Michele R. Cataudella, La Spezia, 2001, pp. 126-127;

Sulla difficoltà di distinguere l'opera della provvidenza da quella del caso e sulla facilità di assegnare all'uno dei due ciò che invece è imputabile alla natura umana o alla saggezza o alla mancanza di senno vd. Plut., comp. Thes. - Rom. 3, 1; Sull. 4,6; Alc. 33, 2; Crass. 27, 6; Tìm. 19, 1.

Plut., aet. Rom. 74

Plut., Aem. 36, 3.

Plut., Nic. 11, 9.

Plut., [cons, ad Apoll.] 104D. 


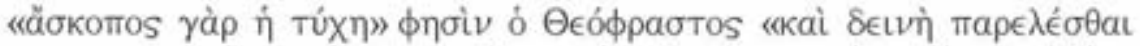

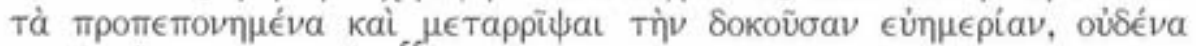

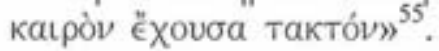

Nelle Vitae Plutarco si interroga a più riprese sull'incidenza che la fortuna ha

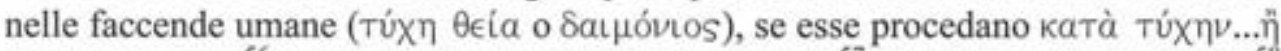

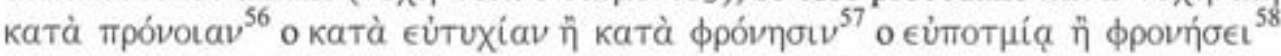
perché la Túxๆ, pur se molto dissimile dalla sapienza è, come scrive Ione di Chio, creatrice di cose ad essa molto simili ${ }^{59}$, cosicché tutto ciò che si può attribuire

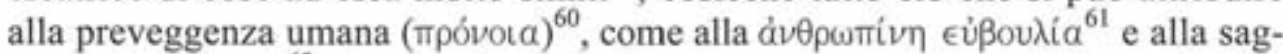
gezza $\left(\phi \rho v^{\prime} \eta \eta \iota s\right)^{62}$ che alla fortuna si contrappongono, è possibile attribuirlo anche alla fortuna ${ }^{63}$.

Nel chiedersi se i «grandi) della storia siano pervenuti al successo nelle loro imprese più grandi con l'aiuto della buona sorte oppure per merito della loro saggezza ${ }^{64}$, Plutarco assegna un ruolo di grande importanza alla fortuna, che ora come divinità benefica e come $\epsilon$ ỦTuxia interviene ad assicurare successi e vittorie, a far prosperare le città, a rendere illustri gli uomini, a elevarli alla celebrità e al potere ${ }^{65}$, ora invece come demone invidioso e vendicativo o come nemesi interviene a sconvolgere e a recidere la loro prosperità. Ma il suo potere non risulta determinante, perché Plutarco tende ad escludere sia l'intervento divino come causa e principio delle azioni e delle

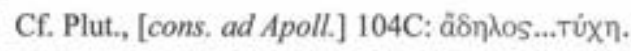

Plut., fort. Rom. 316E . Sulla mpóvota come previdenza umana e sinonimo di €ủßouגía ed áp€тí

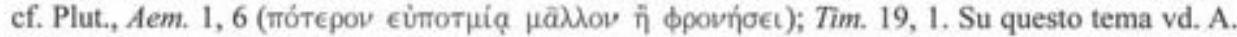
BarigazzI, "Plutarco e il corso futuro della storia", Prometheus 1984, p. 270: «Nell'orazione (sc.

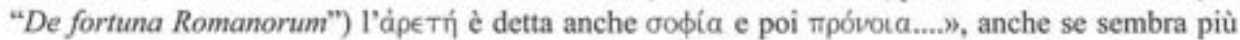
propenso a interpretare прóvot $\alpha$ come "Provvidenza" divina che non come "previdenza" umana; G. ForN1, Plutarco, La fortuna dei Romani, op. cit., p. 102 n. 6: «ll dilemma fortuna o virtù è mutato da Plutarco in fortuna o previdenza, forse per amore di variazione, con la sostituzione della Pronoia all'Areten.

Plut., Tim. 1, 6.

Plut., Aem. 1, 6.

Plut., fort. Rom.316D.

Plut., fort. Rom. 316E.

Plut., fort. Rom. 322A.

Plut., Tim. 1, 6 .

Plut., Tim. 19, 1.

Plut., Tim. 19, 1 /21, 5; Aem. 1, 6/ 12, 2; Alex. 20, 7, 58, 2 ; Phoc. 1, 6; Sert. 1, 1, 10, 6; Sull. 6, 5 ; Dion, 2, 1 ; comp. Arist. - Cat. Ma. 2, 5; fort. Rom. 316C, 316EF, 317C, 318D, 320AB; tranq. an. 475CD; an corp. affect. 500C; laud. ips. 542EF; ser mum. vind. 557B; gen. Socr. 575; Her, mal. $856 \mathrm{~B}$.

Plut., fort. Rom. 316D. 
realizzazioni umane, sia la Túx $\eta$ come causa di virtù e di vizi. Fu infatti la virtù a rendere grande Romolo ${ }^{66}$ come Alessandro e la più meravigliosa delle opere umane, l'impero romano, pur se sollecitato dalla scorta e dal soffio divino della fortuna ${ }^{67}$, non fu portato a compimento dalla €UंTuxía, ma dalla preveggenza e dalla virtù umana $^{68}$. Esso fu il risultato non della fortuna, ma della virtù fortunata (ảp€Tì $\epsilon \dot{U}-$

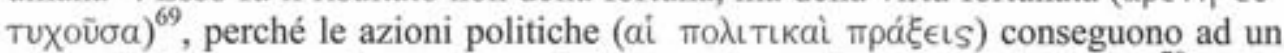
tempo bellezza e grandezza solo se la fortuna è congiunta con la $\phi \rho o ́ v \eta \sigma s^{70}$.

Come l'intervento divino può destare $\mathrm{o}$, al contrario, bloccare la nostra facoltà di agire o di decidere ${ }^{71}$ per mezzo di idee e pensieri iniziali, ma non è in grado di oscurare il $\tau \rho$ ó$^{\prime} \mathrm{S}^{72}$, soprattutto quand'esso è consolidato e rafforzato dalla ragione e dallo studio della filosofia ${ }^{73}$, cioè quand'esso ha raggiunto la fermezza ( $\epsilon \dot{\text { - }}$ -

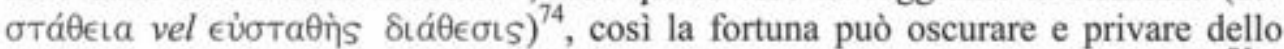

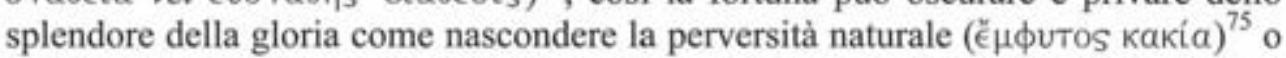
rivelare, come nel caso di Sertorio, la malvagità di una natura che si ammanta di virtù $^{76}$, ma non può privare della virtù chi ha ricevuto un'educazione degna di un uomo libero, come dimostrò Focione che aveva seguito all'Accademia le lezioni di Platone prima e di Senocrate poi ${ }^{77}$.

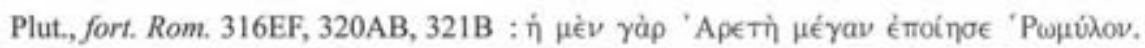
Plut., fort. Rom. 323EF.

Plut., fort. Rom. 323A ; Crass. 26, 9.

Plut., Tim. 21, 5, 36, 4-5 ; H. G. IngenKAmP, "Apetì єu்tuxoũoa (Plutarch, Tim. 36) und die Last der Leichtigkeit", Rh. Mus. Band Heft 1, 1997, pp. 71-89. Sulla limitazione dell'intervento divino cf. Plut., Cor. 32, 5; Fab. 17, 1; ser. mum. vind. 549BC.

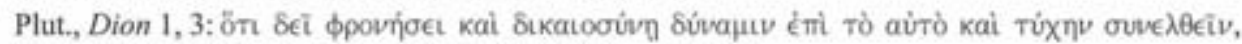

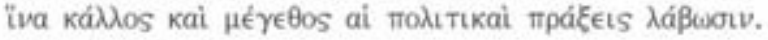

Plut., Cor: 32, 8.

Plut., fort. Rom. 323B.

Plut., Tim. 6, 1.

Plut., cons, ad wxor: 608D, 611A.

Plut., Arat. 49, 1.

Plut., Sert. 10, 6-7. L' opposizione, a mio avviso, non riguarda la natura di una persona (physis)

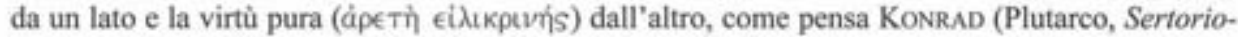
Eumene, Milano 2004, pp. 244-246 n. 80) - per la quale l'intellettuale di Cheronea avrebbe fatto ricorso a delle voces technicae quali quelle di "virtù perfetta" e "virtù imperfetta" o "virtù naturale" - ma investe "la virtù genuina, fondata sulla ragione e sull'educazione" da un lato e dall'altro la "finzione di virtù" o "parvenza di virtù" che, con il mutare del destino, si rivela come stoltezza e malvagità latente, indipendentemente dal possesso di buone qualità naturali (vd. Plut., Sert. 10,

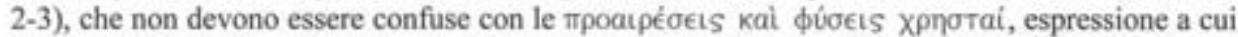
l'intellettuale ricorre per indicare il carattere virtuoso di una persona (Plut., Sert. 10, 6). Su questo tema vd. C. GIL., "The Question of carachter-development : Plutarch und Tacitus", CQ, 33 (1983) 469-487, in particolare pp. 479-481.

Plut., Phoc, 4, 2. 


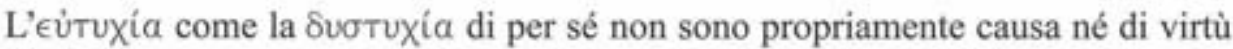
né di vizi, ma creano piuttosto le condizioni che permettono o di dissimulare o di

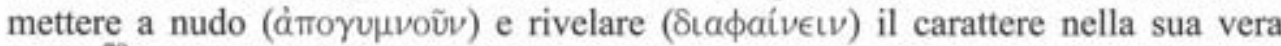

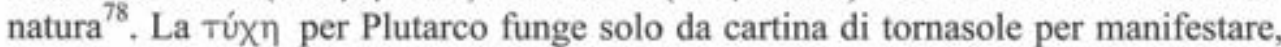
alla stessa stregua di un liquido che mette alla prova l'integrita dei vasi ${ }^{79}$, la vera indole di un uomo, se essa sia provvista di $\pi \alpha t \delta \in i a$ come quella di Eumene ${ }^{80} \mathrm{o}$ se sotto una parvenza e finzione di virtù si nasconda un'indole malvagia ${ }^{81}$, che si scopre allora perché ha la possibilità di farlo, come nel caso di Pisistrato, Alcibiade, Sertorio e Silla ${ }^{82}$. Infatti i mutamenti completi di carattere come quello di Filippo ${ }^{83}$ o di Silla non devono considerarsi per Plutarco come un'evoluzione o un'alterazio-

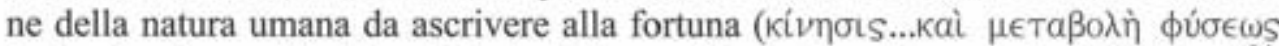

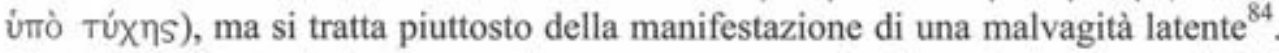
Non è quindi tanto la buona o cattiva sorte a causare le alterazioni e le $\mu \in \tau a \beta 0 \lambda a i ́$ del carattere, quanto l'áớ́veta e l'ảtovía della facoltà razionale dell'anima, che in particolari condizioni non è più in grado di controllare la passionalità.

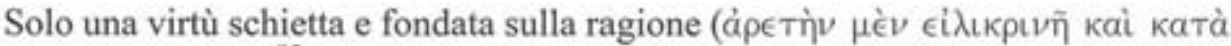

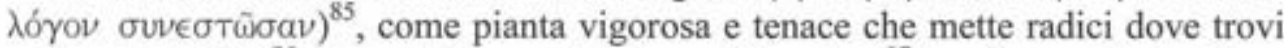
un terreno adatto ${ }^{86}$, non subisce profonde modificazioni ${ }^{87}$, trasformandosi nel suo contrario. Essa non si lascia corrompere o indebolire dalla üßpıs nella buona sorte né abbattere dalle avversita ${ }^{88}$, perché solo chi ha un male dentro di sé, dovuto all'

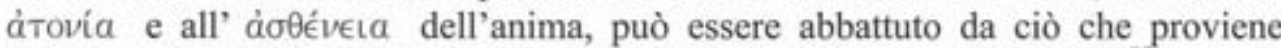
dall'esterno. La falsa o vana opinione infatti è sempre fonte di dolore ${ }^{89}$, mentre l'insegnamento filosofico, che traspare nelle azioni di chi è stato educato ${ }^{90}$, grazie

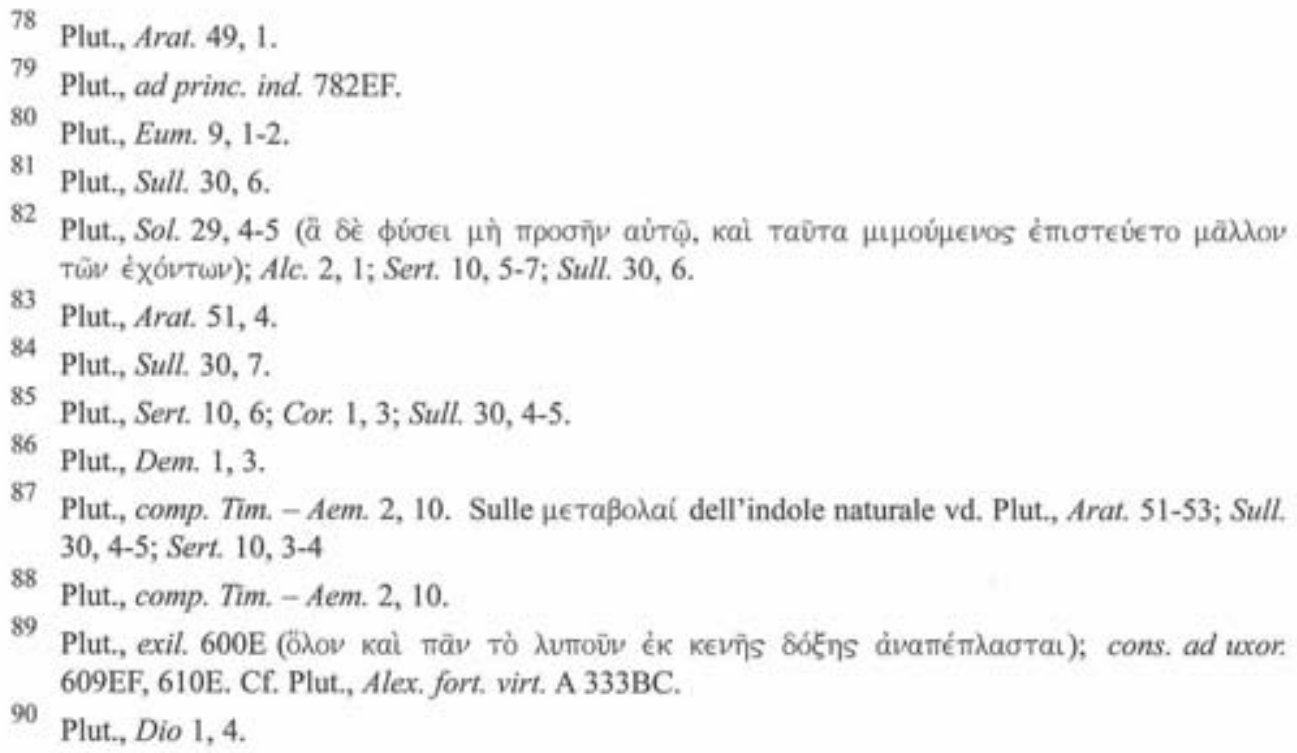




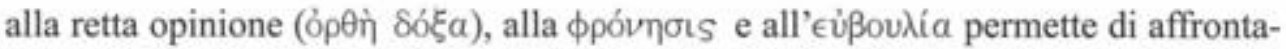
re con moderazione le sventure, il dolore, $\mathrm{i}$ lutti e persino la morte.

Ma il Cheronese, pur ribadendo che uno spirito integralmente virtuoso non si lascia guastare dalla tracotanza nella buona sorte, né abbattere dalle sventure, come dimostrò tra gli altri Emilio Paolo ${ }^{91}$, che con la forza della ragione riusci a dominare le passioni dell'animo e a mantenere sempre un comportamento dignitoso anche in un forte dolore, quale quello per la perdita dei figli ${ }^{92}$, tuttavia ammette, in accordo con l'Etica del peripatetico Teofrasto ${ }^{93}$, la possibilità che anche un carattere virtuoso, soprattutto quando sia immeritatamente oltraggiato ${ }^{94}$, subisca alterazioni

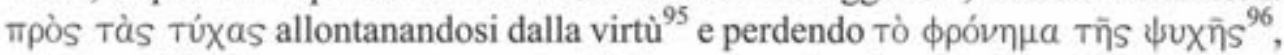

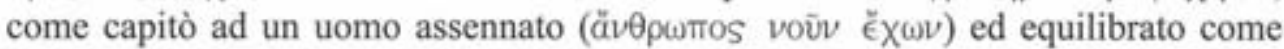
Pericle, che si era sempre distinto per moderazione, mitezza d'animo e altezza di pensiero ${ }^{97}$, quando perse Paralo, l'ultimo dei suoi figli legittimi ${ }^{98}$, e quando fu colpito dalla peste ${ }^{99}$ o come accadde a Timoleonte che non seppe dominare con la forza della ragione il dolore che provò per l'uccisione del fratello Timofane ${ }^{100}$, e ad Alessandro, il "filosofo in azione" 101 , quando presero a calunniarlo, attaccandone la reputazione ${ }^{102}$. Nella vita di Solone ${ }^{103}$ Plutarco scrive che «anche la virtù, di cui non c'è bene né più grande né più piacevole, noi la vediamo crollare a causa di malattie e farmaci», cosa che anche Crisippo, pur contraddicendosi, fini per riconoscere nel

Plut., comp. Tim. - Aem. 2, 10; Sert. 10, 6; Phoc, 1, 6.

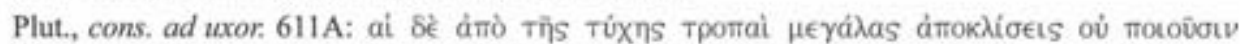

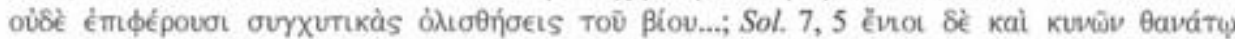

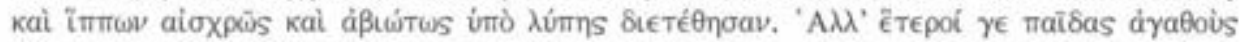

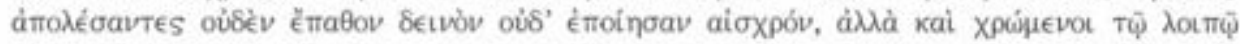

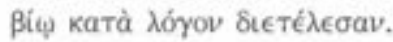

93 Plut., Per. 38, 2. Vd. Cic., Tusc. disp. V 9, 25: Possum igitur, cui concesserim in malis esse dolores corporis, in malis naufragia fortunae, huic suscensere dicenti non ommis bonos esse beatos, cum in

100

101

102 103 omnis bonos ea quae ille in malis mumerat cadere possint?; Plut., [cons. ad Apoll.] 104D.

Plut., Sert. 10, 6; Aem. 26, 9-10.

Plut., Sol. 7, 2; Phoc. 1, 6; Per.36, 8.

Plut., Per: 36,$8 ; 38,1$.

Plut., Per: 39, 1-2.

Plut, Per, 36, 8-9.

Plut., Per. 38, 2.

Plut., Tim. 4, 8-5, 4 .

Plut., Alex. 42, 3-4. Sulla fortuna che, quando contende con nomini di valore, ha una forza sufficiente a indebolire la loro reputazione di virtù vd. Plut., Phoc. 1, 6.

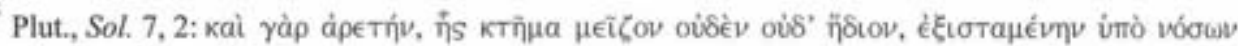
кaì фарца́kwv ópōjev. 
sesto libro delle sue Ricerche etiche ${ }^{104}$.

Se dal piano storico si passa a quello filosofico, molto piccola è per Plutarco la parte dell'uomo esposta agli attacchi della Túx $\eta$, considerato che l'unico bersaglio ch'egli offre alla sorte è il corpo, mentre resta padrone della parte migliore di sé, in cui risiedono $\mathrm{i}$ beni più grandi ${ }^{105}$. La fortuna può colpirlo quindi con una malattia, privarlo delle ricchezze, metterlo in cattiva luce presso il popolo o il tiranno, ma non potrà mai far diventare malvagio un uomo buono, coraggioso e magnanimo, né privarlo della sua disposizione interiore ${ }^{106}$, che è la sorgente della felicità ${ }^{107}$. Per il biografo delle Vitae come per il filosofo dei Moralia la vera felicità dipende in massi-

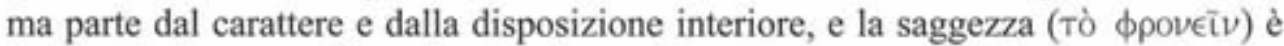

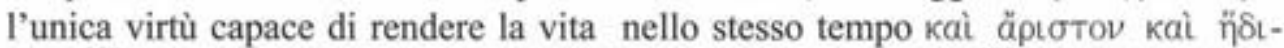

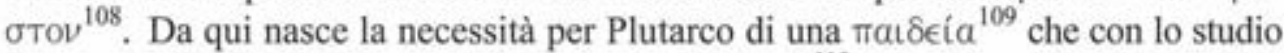
della filosofia consolidi e rafforzi il giudizio dell'uomo ${ }^{110}$ eliminando la debolezza dell'anima, che produce dolori senza limiti e paure in uomini non preparati dalla ragione a sostenere le avversità della sorte, e rendendo retto e nobile l'agire umano ${ }^{\text {III }}$.

Per quanto riguarda la nozione di TúX $\eta$ esiste dunque un unico filo conduttore che lega i Moralia alle Vitae, che già nel XVI secolo non era sfuggito al traduttore ed editore di Plutarco, Guglielmo Xylander (Wilhelm Holtzman, 1532 - 1576) che nella "Vita di Plutarco" («Vita Plutarchi e Xylandro»), premessa al primo volume, contenente le Vitae, dell'edizione Stephaniana II o Francofurtana del $1599^{112}$, scrive che Plutarco compose le Vitae "non verbis sed rebus", riportando "non unius temporis aut populi tantum, sed diversorum exempla» per ammonirci «...ne casum in rebus humanis plus quam consilium posse iudicemus: neve tritum illud nostro assensu comprobemus

\footnotetext{
${ }^{104}$ Plut., Stoic. rep. 1046F-1047A.

${ }^{105}$ Plut., tranq, an. $475 \mathrm{CD}$.

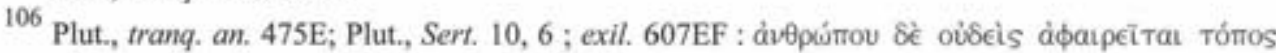

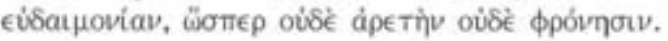

${ }^{107}$ Plut., tranq. an. 467A; coh. ira 464A.

108 Plut., trang. an. $466 \mathrm{~F}-467 \mathrm{~A}=$ Dem. $1,1$.

109 Sull'importanza della paideia per plasmare un carattere secondo virtù vd. Plut., Dio 10, 1-5.

${ }^{110}$ Plut., Tìm. 6, 1. Cf. Plut., Sert. 10,6.

111 Plut., Sol. 7, 5-6; Ant. 17, 4 ; Dio 1, 4; Alex. fort. virt. A 333BC. Cf. Plut., [cons. ad Apoll.] 103F:

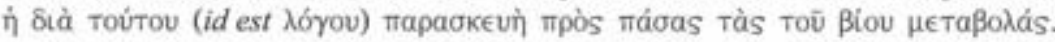

112 Plutarchi Chaeronensis quae exstant omnia cum latina interpretatione Hermanni Cruseri et Guilielmi Xylandri et doctorum virorum notis et libellis variantium lectionum ex Mss. Codd. diligenter collectarum et indicibus accuratis. Francofurti apud Andreae Wecheli heredes, Claudium Marnium et Ioannem Aubrium, M.D.XCIX.
} 
«Vitam regit fortuna, non sapientia»,

suggerendo indirettamente quella che io giudico essere la corretta interpretazione

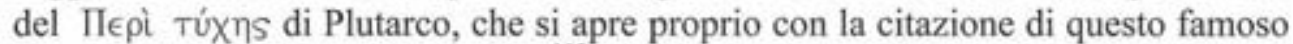
verso del poeta tragico Cheremone ${ }^{113}$

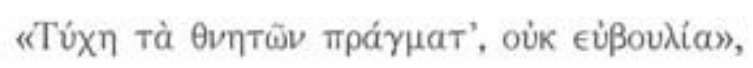

già riportato a mo' di sententia nel V libro delle Tusculanae disputationes ${ }^{114}$, in cui l'Arpinate riferisce tra l'altro delle aspre critiche che nelle opere e nelle lezioni di tutti i filosofi erano state mosse allo scolarca del Peripato, Teofrasto, che definisce elegantissimus omnium philosophorum et eruditissimus ${ }^{115}$ per avere scritto nel De

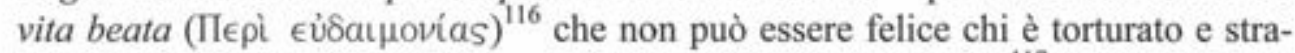
ziato («is qui torqueatur, qui crucietur, beatus esse non possit») ${ }^{117}$ e poi per avere approvato nel Callisthenes sive de luctu ${ }^{118}$ illam sententiam «vitam regit fortuna, non sapientian, di cui nessun filosofo ebbe a dire mai nulla di più fiacco ${ }^{119}$.

Questa concordanza con le Tusculanae ha finito per condizionare l'interpretazione dello scritto plutarcheo sin oltre la metà degli anni ' 50 . Tutti gli studiosi che nel corso dell'ultimo decennio del XIX secolo [Dümmler (1889), Heinze (1890), Giesecke (1891), Schlemm (1893), Siefert (1896), Dyroff (1897)] e nella prima metà del XX secolo [Buriks (1950), Ziegler (1951)] si sono occupati di questo scritto hanno concordemente fatto propria l'ipotesi formulata nel 1889 da Dümmler in un articolo dal titolo "Ein stoischer gegner Theophrasts", cioè che P. riferisse nel

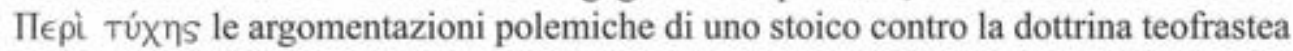
della $€$ Ủbaıนovia, salvo poi dividersi sul nome del filosofo stoico (Aristone di Chio, contemporaneo di Cleante, lo scolarca Zenone, l'alunno di Cleante, Sfero, autore

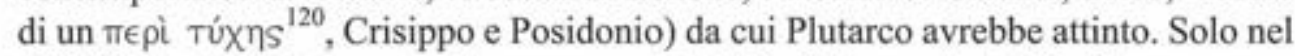
1969 Babut, nel riconoscere la presenza indubbia di elementi stoici, rilevava come essi rappresentassero comunque "des lieux communs dont l'origine tend à s'estomper» e metteva in guardia dal dedurne un adeguamento di Plutarco all'ideologia

\footnotetext{
${ }^{113}$ Chaerem. $71 \mathrm{~F} 2$ Snell $=\operatorname{Tr} G F 1217$.

${ }^{114}$ Cic., Tusc. V 23-25 = Thphr., Fr. L 53 Fortenbaugh.

${ }^{115}$ Cic., Tusc., V 9, 24; Acad. 133 vir et oratione suavis et ita moratus, ut prae se probitatem quandam et ingenuitatem ferat.

${ }^{116}$ Cf. D. L., V 43.

${ }^{117}$ Cic., Tusc. V 9, 24. A questo proposito Cicerone nega che Teofrasto nel de vita beata abbia usato l'espressione in rotam... beatam vitam non escendere.

${ }^{118}$ Cf. Cic., Tusc. III 10, 21.

119 Cf. Cic., Acad. 1 33, 35 = Thphr., Fr. L 57 Fortenbaugh: spoliavit...virtutem suo decore imbecillamque reddidit quod negavit in ea sola positum esse beate vivere. 120 SVF I $620=$ D. L., VII 178.
} 
stoica $^{121}$. A Babut ha poi fatto eco nel 1989 il connazionale Klaerr, l'editore fran-

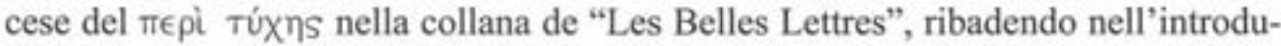
zione questa tendenza e precisando che l' utilizzazione di temi stoici ' $n$ 'implique nullement une adhésion, même momentanée, de Plutarque à la doctrine de Portique" ${ }^{22}$. Questi studi hanno di fatto aperto la strada alla innovativa e, per certi aspetti, rivoluzionaria interpretazione di Barigazzi, che giudica la difesa del valore della virtù e del logos contro l'instabilità della fortuna non polemicamente diretta contro il Peripato, bensi "non disforme...dai principi etici del Peripato"123.

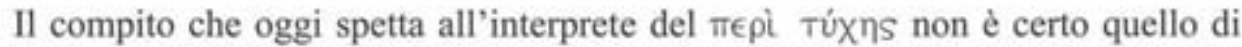
individuare la fonte stoica o peripatetica, dalla quale Plutarco avrebbe attinto, ma quello di giustificare la consistente presenza di temi stoici, che non può essere liquidata semplicemente come una ripresa di "luoghi comuni", e ad un tempo quello di verificare in concreto l'attendibilità dell'ipotesi interpretativa avanzata da Barigazzi, che resta tutta da dimostrare, nella convinzione che nessuna delle due tendenze interpretative sino ad oggi formulate sia depositaria dell'intera verità, ma che in ognuna si nasconda una parte di verità.

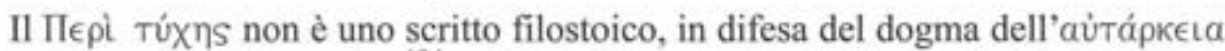

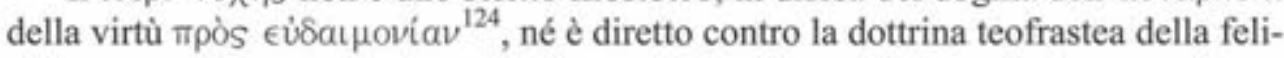
cità, come del resto non è antiteofrasteo il De Alexandri Magni virtute aut fortu-

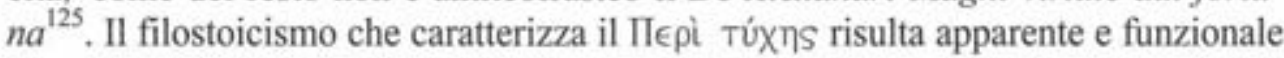
alla strategia di colpire gli Stoici sul loro stesso terreno.

Plutarco nel П€ gli affari umani, come recita il trimetro di Cheremone, diventa di conseguenza inevitabile attribuire alla fortuna tutte le azioni che sono proprie delle arti e delle virtù

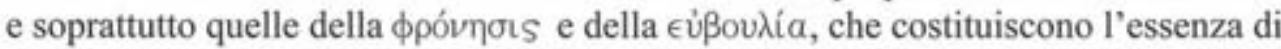
ogni virtù e sono prerogativa (iઠ̋ıv é $\rho \gamma o \nu$ ) dell'essere umano.

121

D. BABut, Plutarque et le Sloĭcisme, Paris 1969, p. 83.

122 Plutarque, De la fortune, Oeuwres Monales I 2. Texte établi et traduit par R. KLAERR, Paris 1989, p. 233.

123 Plutarco, Se la virtì si debba insegnare. Testo critico, introduzione, traduzione a cura di A. BArigazZ, Napoli 1993, p. 53.

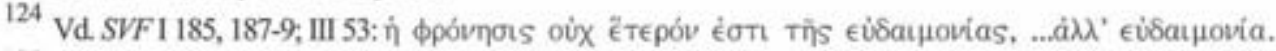

125 Per quanto riguarda il De Alex. Magni virt. aut fort. non condivido l'interpretazione di GRIL.L (Alessandro e Filippo nella filosofia ellenistica e nell 'ideologia politica romana, in M. Sordi (Ed.). Alessandro Magno tra storia e mito, Milano 1984, pp. 123-153; Le polemiche filosofiche di Plutarco, in L. GALLO - R. LAURENTI (Edd.), Strumenti per la ricenca plutanchea, I, Napoli 1992 , pp. 61-78), mentre sono d'accordo con quanto scrive D'ANgelo (Plutarco, La fortuna o la virtì di Alessandro Magno. Prima orazione, Napoli 1998, p. 175): "Il dibattito culturale sorto attomo alla figura di Alex., considerato un tiranno ingiustamente favorito dalla Fortuna, è senza dubbio di provenienza stoica". Una conferma può venire da Sen., Ep. 83, 19;94, 62; 112, 29. 
A questo punto è sufficiente chiedersi chi possano essere quei filosofi contro cui polemizza Plutarco, che tolgono ogni fondamento ad un discorso propriamente etico. La risposta è agevole: le moderne scuole di pensiero, da un lato gli Epicurei

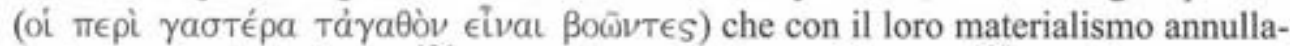
no la «uxí e la $\phi \rho o ́ v \eta \sigma ı S^{126}$, ponendo, per dirla con Cicerone ${ }^{127}$, ogni bene e ogni

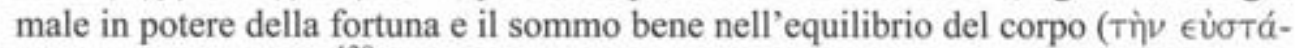

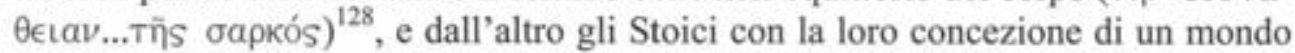
rigorosamente e razionalmente predeterminato, inconciliabile con la moralità e quindi con la libertà dell'uomo.

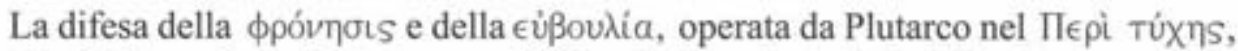
con la riaffermazione della responsabilità e della volontarietà dell'agire umano per

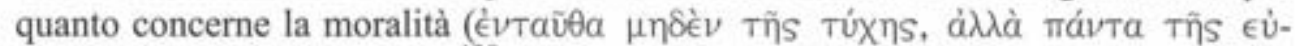

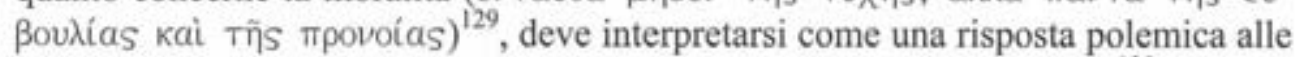
moderne scuole di pensiero, che lungi dall'eliminare il caso e la fortuna ${ }^{130}$, finiscono coll'ammettere che tutto nella vita umana accade secondo una naturale ed immutabile connessione di cause ed effetti, cui si può dare indifferentemente il nome di

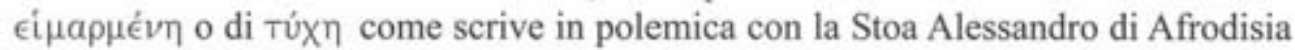

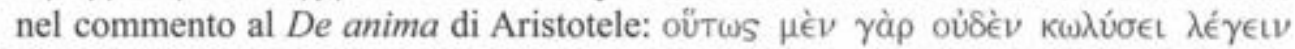

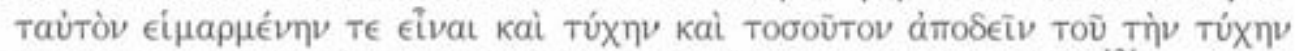

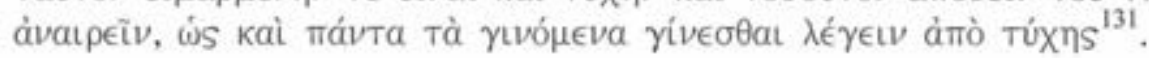

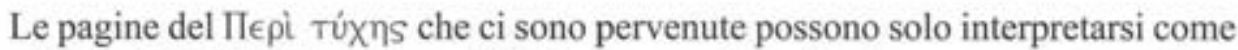
una difesa della libertà e responsabilità umana, minacciata da una concezione di un mondo rigorosamente e razionalmente predeterminato, in cui l'agire umano ( $\tau \grave{a}$

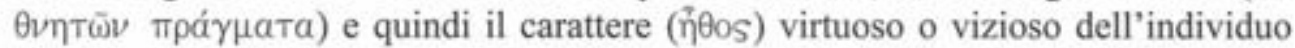
sembra essere il risultato della fortuna e del caso (Tò aủTó $\mu \alpha \tau o \nu$ ) piuttosto che

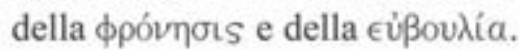

A completare questo quadro non ci resta che aggiungere da un lato quanto sullo stesso tema scrive polemicamente Plutarco nel cap. XLVII del de Stoicorum repugnantiis, e dall'altro l'interrogativo che nel De fato si pone Alessandro di Afrodisia dinanzi ad una concezione che pone la vita umana in balia della Túxๆ.

${ }^{127}$ Cic., Tusc. V 26, 73: cum sit omne et bonum eius (sc. Epicuri) et mali in potestate fortunae.

128 Plut., suav. viv. Epic. 1089D, 1090A, D; adv. Col. 1117A, 1118E, 1125A; tuend. san. 135C. Su

129 Plut., fort. 98F. Cf. ibid. $98 \mathrm{~A}$. questo tema vd. J. Boulogne, Plutarque dans le miroir d'Épicure, Lille 2003, p. 155 n. 26.

${ }^{130}$ Cf. Plut., Stoic. rep. 1045C, 1056AB. Vd. Plutarque, Sur les contradictions stoliciennes, Oeuvres Morales XV, Ire partie. Texte établi par M. CASEVITZ et traduit et commenté par D. BABUT, Paris 2004, pp. 223-4 nu. 302-3.

${ }^{131}$ Alex. Aphr., de fato, (Suppl. Arist. II 2, ed. I. Bruns, Berolini 1892), p. 173, 23-26. 
Plutarco nel de Stoicorum repugnantiis scrive che se si ammette il fato, come intendono gli Stoici, ne consegue che non dipendono da noi né la virtù né la malvagità né l'agire rettamente ${ }^{132}$. Alessandro di Afrodisia, convinto come Plutarco che

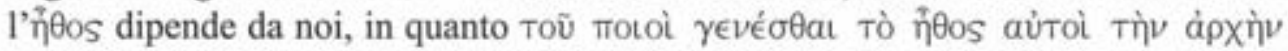

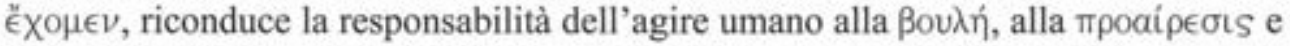

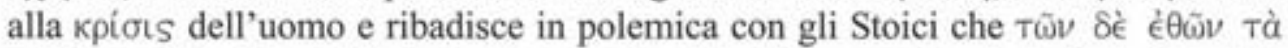
$\pi \lambda \epsilon \tilde{\tau} \sigma \tau \alpha$ '̇ $\phi$ ' $\dot{\eta} \mu \mathrm{i} \nu$, chiedendosi come si potrà affermare che dipendano da noi la virtù e il vizio se si ammette che la Túxๆ governa gli affari umani ${ }^{133}$.

132 Alex. Aphr., de anima (Suppl. Arist. II, 1, cit., pp. 174, 9-11e 175, 9-12.

${ }^{133}$ Cf. Alex. Aphr., de fato (Suppl. Arist. II, 2 cit.), pp. 187, 24-5, 199, 7 ss., 209, 28-210, 3. 
(Página deixada propositadamente em branco) 


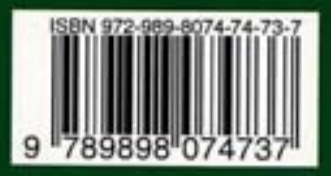

